\title{
On Strict Lyapunov Functions for Rapidly Time-Varying Nonlinear Systems
}

\author{
Frédéric Mazenc, Michael Malisoff, and Marcio S. de Queiroz
}

\begin{abstract}
We explicitly construct Lyapunov functions for rapidly time-varying nonlinear systems. The Lyapunov functions we construct are expressed in terms of oftentimes more readily available Lyapunov functions for the limiting dynamics which we assume are globally asymptotically stable. This leads to new sufficient conditions for global exponential, global asymptotic, and input-to-state stability of fast time-varying dynamics. We apply our results to two examples.
\end{abstract}

Index Terms - time-varying systems, input-to-state stability, Lyapunov function constructions

\section{INTRODUCTION}

The stabilization of nonlinear nonautonomous systems and the construction of their Lyapunov functions are challenging problems that are of significant ongoing interest [8], [10], [17], [18]. One popular approach to guaranteeing stability of nonautonomous systems is the so-called averaging method in which exponential stability of an appropriate autonomous system implies exponential stability of the original dynamics when the time variation is sufficiently fast. See [7] for related results and [11] where the fast time-varying hypothesis is replaced by a homogeneity condition.

The preceding results were extended to more general rapidly time-varying systems of the form

$$
\dot{x}=f(x, t, \alpha t), \quad x \in \mathbb{R}^{n}, t \in \mathbb{R}, \alpha>0
$$

in [12], where the (local) exponential stability of (1) was proven for large constants $\alpha>0$ under appropriate regularity conditions, assuming that a suitable limiting dynamic

$$
\dot{x}=\bar{f}(x, t)
$$

for (1) is exponentially stable. This generalized a result from [6, pp. 190-5] on a restricted class of systems (1) satisfying certain periodicity or almost periodicity conditions. (See also [13] for related results for slowly time-varying systems.) The main arguments of [12] use (partial) averaging but do not lead to explicit Lyapunov functions for (1).

In this note, we pursue a very different approach. Instead of averaging, we explicitly construct a family of Lyapunov functions for (1) that are expressed in terms of more readily available Lyapunov functions for the limiting dynamics (2), which we again assume is asymptotically stable. In addition,

This work was supported by NSF/DMS Grant 0424011.

F. Mazenc is with Projet MERE INRIA-INRA, UMR Analyse des Systèmes et Biométrie INRA, 2 pl. Viala, 34060 Montpellier, France mazencahelios. ensam. inra. fr.

M. Malisoff is with the Department of Mathematics, Louisiana State University, Baton Rouge, LA 70803, USA malisoffelsu . edu.

M.S. de Queiroz is with the Department of Mechanical Engineering, Louisiana State University, Baton Rouge, LA 70803, USA dequeiroz@me. Isu.edu. while [12] assumes (2) is exponentially stable, we allow cases where (2) is merely uniformly globally asymptotically stable (UGAS), in which case we conclude that (1) is UGAS (but not necessarily exponentially stable) when $\alpha>0$ is sufficiently large. While global exponential and global asymptotic stabilities are equivalent for autonomous systems under a coordinate change in certain dimensions, the coordinate changes are not explicit and so do not lend themselves to explicit Lyapunov function constructions [5].

In particular, we show that assumptions similar to those of [12, Theorem 3] imply that (1) is globally (rather than merely locally) exponentially stable; our Lyapunov function constructions are new even in this particular exponential stability case, and our results complement [12]. Moreover, the Lyapunov functions we construct are also input-to-state stable (ISS) or integral ISS Lyapunov functions for the rapidly time-varying control system

$$
\dot{x}=f(x, t, \alpha t)+g(x, t, \alpha t) u
$$

under appropriate conditions on $f$ and $g$; see Remark 12.

In Section II, we provide the relevant definitions and lemmas. In Section III, we present our sufficient conditions for global asymptotic and exponential stability of (1), and for the stability of (3), in terms of appropriate limiting dynamics (2). This leads to our explicit construction of Lyapunov functions for (1) and (3) in terms of our Lyapunov functions for (2), in Corollary 10. We prove the sufficiency of our criteria in Section IV, and we illustrate our results in Section V using two examples. We close in Section VI by summarizing our findings.

\section{ASSUMPTIONS, DEFINITIONS, AND LEMMAS}

We study (1) (which includes dynamics (2) with no $\alpha$ dependence, as special cases) in which we always assume $f$ is continuous in time $t \in \mathbb{R}:=(-\infty,+\infty)$, continuously differentiable $\left(C^{1}\right)$ in $x \in \mathbb{R}^{n}$, null at $x=0$ meaning

$$
f(0, t, \alpha t)=\bar{f}(0, t)=0 \quad \forall t \in \mathbb{R}, \alpha>0
$$

and forward complete, i.e., for each $\alpha>0, x_{o} \in \mathbb{R}^{n}$, and $t_{o} \in \mathbb{R}_{\geq 0}:=[0, \infty)$ there exists a unique trajectory $\left[t_{0}, \infty\right) \ni$ $t \mapsto \phi\left(t ; t_{o}, x_{o}\right)$ for (1) (depending in general on the constant $\alpha>0$ ) that satisfies $x\left(t_{o}\right)=x_{o}$. We set $\mathbb{N}=\{1,2,3, \ldots\}$.

We use the following additional definitions. We say that $N: \mathbb{R}_{\geq 0} \rightarrow \mathbb{R}_{\geq 0}$ is of class $\mathcal{M}$ and write $N \in \mathcal{M}$ provided

$$
\lim _{\eta \rightarrow+\infty} \eta N(\eta)=0 .
$$

A continuous $\delta: \mathbb{R}_{\geq 0} \rightarrow \mathbb{R}_{\geq 0}$ is positive definite provided it is zero only at zero. A positive definite $\delta$ is of class $\mathcal{K}$ 
(written $\delta \in \mathcal{K}$ ) provided it is strictly increasing; if $\delta$ is also unbounded, then we say $\delta$ is of class $\mathcal{K}_{\infty}$ and write $\delta \in \mathcal{K}_{\infty}$. A continuous $\beta: \mathbb{R}_{\geq 0} \times \mathbb{R}_{\geq 0} \rightarrow \mathbb{R}_{\geq 0}$ is of class $\mathcal{K} \mathcal{L}$ (written $\beta \in \mathcal{K} \mathcal{L}$ ) provided (a) $\beta(\cdot, t) \in \mathcal{K}_{\infty}$ for all $t \geq 0$, (b) $\beta(s, \cdot)$ is nonincreasing for all $s \geq 0$, and (c) for each $s \geq 0$, $\beta(s, t) \rightarrow 0$ as $t \rightarrow+\infty$. We always assume there is a $\rho \in \mathcal{K}_{\infty}$ such that $|f(x, t, \alpha t)| \leq \rho(|x|)$ everywhere.

We next define our stability properties for (2) but the same definitions apply for (1) for any fixed choice of the constant $\alpha>0$. We say that (2) is uniformly globally asymptotically stable $(U G A S)$ provided there exists $\beta \in \mathcal{K} \mathcal{L}$ such that

$$
\left|\phi\left(t ; t_{o}, x_{o}\right)\right| \leq \beta\left(\left|x_{o}\right|, t-t_{o}\right) \text { if } t \geq t_{o} \geq 0, x_{o} \in \mathbb{R}^{n}
$$

where $|\cdot|$ is the usual Euclidean norm and $\phi$ is the flow map for (2), and we call (2) uniformly globally exponentially stable (UGES) provided there exist constants $D>1$ and $\lambda>0$ such that (6) is satisfied with the choice

$$
\beta(s, t)=D s e^{-\lambda t} .
$$

Recall that (2) is UGAS if and only if it has a (strict) Lyapunov function, i.e., a $C^{1}$ function $V$ that admits $\delta_{3} \in \mathcal{K}$ and $\delta_{1}, \delta_{2} \in \mathcal{K}_{\infty}$ such that for all $t \in \mathbb{R}_{>0}$ and $\xi \in \mathbb{R}^{n}$,

(L1) $\delta_{1}(|\xi|) \leq V(\xi, t) \leq \delta_{2}(|\xi|)$ and

(L2) $V_{t}(\xi, t)+V_{\xi}(\xi, t) \bar{f}(\xi, t) \leq-\delta_{3}(|\xi|)$,

where subscripts on $V$ denote partial gradients [2]. When (2) is also UGES, the proof of [7, Theorem 4.14] shows:

Lemma 1: Assume (2) satisfies the UGES condition (6)(7) for some constants $D>1$ and $\lambda>0$ and that there exists $K>\lambda$ such that $|(\partial \bar{f} / \partial \xi)(\xi, t)| \leq K$ for all $\xi \in \mathbb{R}^{n}$ and $t \in \mathbb{R}_{\geq 0}$. Then (2) admits a Lyapunov function $V$ and constants $c_{1}, c_{2}, c_{3}>0$ such that

$$
\begin{aligned}
& c_{1}|\xi|^{2} \leq V(\xi, t) \leq c_{2}|\xi|^{2}, \quad\left|V_{\xi}(\xi, t)\right| \leq c_{3}|\xi| \\
& \text { and } V_{t}(\xi, t)+V_{\xi}(\xi, t) \bar{f}(\xi, t) \leq-|\xi|^{2}
\end{aligned}
$$

hold for all $t \in \mathbb{R}_{\geq 0}$ and $\xi \in \mathbb{R}^{n}$.

Remark 2: We can choose the constant $c_{3}$ in (8) to be

$$
c_{3}=\frac{4 D(\Theta-1)}{(K-\lambda)}, \text { where } \Theta=(\sqrt{2} D)^{K / \lambda-1} .
$$

This also follows from the proof of Lemma 1 in [7].

Motivated by Lemma 1, we find it convenient to use the following compatibility condition for UGAS systems (2):

Definition 3: Given $\delta \in \mathcal{K}$, the dynamics (2) is called $\delta$ compatible provided it admits a Lyapunov function $V \in C^{1}$ and two constants $\bar{c} \in(0,1), \overline{\bar{c}}>0$ such that:

P1) $V_{t}(\xi, t)+V_{\xi}(\xi, t) \bar{f}(\xi, t) \leq-\bar{c} \delta^{2}(|\xi|) \quad \forall \xi, t$;

P2) $\left|V_{\xi}(\xi, t)\right| \leq \delta(|\xi|)$ and $|\bar{f}(\xi, t)| \leq \delta(|\xi| / 2) \quad \forall \xi, t$; P3) $\delta(s) \leq \overline{\bar{c}} s \quad \forall s \geq 0$.

Remark 4: Note the asymmetry in the bounds on $\left|V_{\xi}\right|$ and $|\bar{f}|$ in P2). One easily checks that if (2) satisfies the assumptions of Lemma 1 , then it is $\delta$-compatible with $\delta(s)=\left(c_{3}+2 K\right) s$. However, by varying $\delta$ (including cases where $\delta$ is bounded), one finds a rich class of non-UGES $\delta$-compatible dynamics as well; see e.g. Section V-A below.
We also consider nonautonomous control systems

$$
\dot{x}=F(x, t, u)
$$

which we always assume are continuous in all variables and $C^{1}$ in $x$ with $F(0, t, 0) \equiv 0$, and whose solution for a given control function $\mathbf{u} \in \mathcal{U}(:=$ all measurable locally essentially bounded functions $[0, \infty) \rightarrow \mathbb{R}^{m}$ ) and given initial condition $x\left(t_{o}\right)=x_{o}$ we denote by $t \mapsto \phi\left(t ; t_{o}, x_{o}, \mathbf{u}\right)$. We always assume (10) is forward complete, i.e., all trajectories $\phi\left(\cdot ; t_{o}, x_{o}, \mathbf{u}\right)$ have domain $\left[t_{o},+\infty\right)$. Later we specialize to the case where (10) has the form (3). We next recall the input-to-state stable (ISS) and integral input-to-state stable (iISS) properties [14], [15]. Let $|\mathbf{u}|_{I}$ denote the essential supremum of $\mathbf{u} \in \mathcal{U}$ restricted to any interval $I \subseteq \mathbb{R}_{>0}$.

Definition 5: (a) We say that (10) is ISS provided there exist $\gamma \in \mathcal{K}_{\infty}$ and $\beta \in \mathcal{K} \mathcal{L}$ for which

$$
\left|\phi\left(t ; t_{o}, x_{o}, \mathbf{u}\right)\right| \leq \beta\left(\left|x_{o}\right|, t-t_{o}\right)+\gamma\left(|\mathbf{u}|_{\left[t_{o}, t+t_{o}\right]}\right)
$$

holds when $t \geq t_{o} \geq 0, x_{o} \in \mathbb{R}^{n}$, and $\mathbf{u} \in \mathcal{U}$. If in addition $\beta$ has the form (7), then we say that (10) is input-to-state exponentially stable (ISES). (b) We say that (10) is iISS provided there exist $\mu, \gamma \in \mathcal{K}_{\infty}$ and $\beta \in \mathcal{K} \mathcal{L}$ such that

$\mu\left(\left|\phi\left(t ; t_{o}, x_{o}, \mathbf{u}\right)\right|\right) \leq \beta\left(\left|x_{o}\right|, t-t_{o}\right)+\int_{t_{o}}^{t_{o}+t} \gamma(|\mathbf{u}(s)|) \mathrm{d} s$

holds when $t \geq t_{o} \geq 0, x_{o} \in \mathbb{R}^{n}$, and $\mathbf{u} \in \mathcal{U}$.

The following Lyapunov function notions agree with the usual ISS and iISS Lyapunov function definitions when (10) is autonomous, because functions $\chi \in \mathcal{K}_{\infty}$ are invertible.

Definition 6: Let $V: \mathbb{R}^{n} \times \mathbb{R}_{\geq 0} \rightarrow \mathbb{R}_{\geq 0}$ be $C^{1}$ and admit $\delta_{1}, \delta_{2} \in \mathcal{K}_{\infty}$ that satisfy (L1) above. (a) We call $V$ an ISS Lyapunov function for (10) provided there exist $\chi, \delta_{3} \in \mathcal{K}_{\infty}$ such that for all $t \in \mathbb{R}_{\geq 0}, \xi \in \mathbb{R}^{n}$, and $u \in \mathbb{R}^{m}$, we have:

$$
|u| \leq \chi(|\xi|) \Rightarrow V_{t}(\xi, t)+V_{\xi}(\xi, t) F(\xi, t, u) \leq-\delta_{3}(|\xi|)
$$

(b) We call $V$ an iISS Lyapunov function for (10) provided that there exist $\Delta \in \mathcal{K}_{\infty}$ and a positive definite function $\nu: \mathbb{R}_{\geq 0} \rightarrow \mathbb{R}_{\geq 0}$ such that

$$
V_{t}(\xi, t)+V_{\xi}(\xi, t) F(\xi, t, u) \leq-\nu(|\xi|)+\Delta(|u|)
$$

for all $t \in \mathbb{R}_{\geq 0}, \xi \in \mathbb{R}^{n}$, and $u \in \mathbb{R}^{m}$.

Note that $\nu$ in (12) need not be of class $\mathcal{K}$. Since (10) has an ISS Lyapunov function when it is ISS (by the argument of [16]), the proof of [1, Theorem 1] shows that if (10) is autonomous and ISS, then it is also iISS, but not conversely, since e.g. $\dot{x}=-\arctan (x)+u$ is iISS but not ISS. The next lemma follows from the arguments of [1], [4], [14], [16].

Lemma 7: If (10) admits an ISS (resp., iISS) Lyapunov function, then it is ISS (resp., iISS).

\section{THEORETICAL RESULT}

We show that the main conditions of [12, Theorem 3] ensure not just local exponential stability of the fast-varying system (1), but also that the system is UGES. In fact, we show the conditions imply

$$
\dot{x}=f(x, t, \alpha t)+u, \quad x \in \mathbb{R}^{n}, u \in \mathbb{R}^{n}
$$


is ISES when $\alpha>0$ is sufficiently large, but see Remark 12 below for results for more general control-affine systems (3). Our main assumption will be the following generalization of [12, Property 2]: There exist $\delta \in \mathcal{K}$, a $\delta$-compatible dynamics (2), and $N \in \mathcal{M}$ (cf. condition (5) above) such that for all $x \in \mathbb{R}^{n}$, all $r \in \mathbb{R}$, and sufficiently large $\eta>0$, we have

$$
\left|\int_{r-\frac{1}{\eta}}^{r+\frac{1}{\eta}}\left\{f\left(x, l, \eta^{2} l\right)-\bar{f}(x, l)\right\} \mathrm{d} l\right| \leq \delta(|x| / 2) N(\eta)
$$

of which [12, Property 2] is the special case where $\delta(s)=2 s$. Two more advantages of our result are that (a) our result also applies to cases where the limiting system (2) is UGAS but not necessarily exponentially stable (cf. Section V-A below) and (b) our proof provides explicit constructions for Lyapunov functions for (13) (cf. Corollary 10 below).

Theorem 8: Consider a system (1). Assume there exist $\delta \in \mathcal{K}$, a $\delta$-compatible UGAS system (2), a constant $\eta_{o}>0$ and $N \in \mathcal{M}$ such that (14) holds whenever $\eta \geq \eta_{o}, x \in \mathbb{R}^{n}$ and $r \in \mathbb{R}$. Assume there is a constant $K>1$ such that:

$$
\begin{aligned}
& \left|\frac{\partial \bar{f}}{\partial x}(x, t)\right| \leq K, \quad\left|\frac{\partial f}{\partial x}(x, t, \alpha t)\right| \leq K, \text { and } \\
& |f(x, t, \alpha t)| \leq \delta(|x| / 2) \quad \forall t \in \mathbb{R}, x \in \mathbb{R}^{n}, \alpha>0 .
\end{aligned}
$$

Then there is a constant $\underline{\alpha}>0$ such that for all constants $\alpha \geq \underline{\alpha}$, (1) is UGAS and (13) is iISS. If in addition $\delta \in \mathcal{K}_{\infty}$, then (13) is ISS for all constants $\alpha \geq \underline{\alpha}$. In the special case where (2) is UGES, (1) is UGES for all constants $\alpha \geq \underline{\alpha}$ and (13) is ISES for all constants $\alpha \geq \underline{\alpha}$.

Remark 9: By (4), the condition $|f(x, t, \alpha t)| \leq \delta(|x| / 2)$ in (15) is redundant when $\delta$ has the form $\delta(s)=\bar{r} s$ for a constant $\bar{r}>0$, since $\bar{r}$ can always be enlarged.

\section{PROOF OF THEOREM 8 AND REMARKS}

We first assume that (2) is UGAS and $\delta \in \mathcal{K}_{\infty}$, and we prove the ISS property for (13) for large $\alpha>0$. In what follows, we assume all inequalities and equalities hold wherever they make sense, unless otherwise indicated. Let $\alpha=\eta^{2}$ with $\eta \geq \eta_{o}, \mathbf{u} \in \mathcal{U}$, and $x(t)$ be a trajectory for (13) and $\mathbf{u}$, with arbitrary initial condition. Set

$$
z(t)=x(t)+R(x(t), t), \quad \text { where }
$$

$$
\begin{aligned}
& R(x, t)= \\
& -\frac{\eta}{2} \int_{t-2 / \eta}^{t} \int_{s}^{t}\left\{f\left(x, l, \eta^{2} l\right)-\bar{f}(x, l)\right\} \mathrm{d} l \mathrm{~d} s .
\end{aligned}
$$

Set $p(t, l)=f\left(x(t), l, \eta^{2} l\right)-\bar{f}(x(t), l)$. With $p$ so defined, one easily checks (via Fubini's Theorem, as was done e.g. in [9], [10]) that for any $\tau>0$ and $t \geq 0$,

$$
\begin{aligned}
& \frac{d}{d t} \int_{t-\tau}^{t} \int_{s}^{t} p(t, l) \mathrm{d} l \mathrm{~d} s= \\
& \tau p(t, t)-\int_{t-\tau}^{t} p(t, l) \mathrm{d} l+\int_{t-\tau}^{t} \int_{s}^{t} \frac{\partial p}{\partial t}(t, l) \mathrm{d} l \mathrm{~d} s \\
& \text { and }\left|\int_{t-\tau}^{t} \int_{s}^{t} p(t, l) \mathrm{d} l \mathrm{~d} s\right| \leq \frac{\tau^{2}}{2} \max _{t-\tau \leq l \leq t}|p(t, l)| .
\end{aligned}
$$

Taking $\tau=2 / \eta$, condition (18) multiplied through by $-\eta / 2$ and $f\left(x(t), t, \eta^{2} t\right)-p(t, t) \equiv \bar{f}(x(t), t)$ and (16) give

$$
\begin{aligned}
\dot{z}(t) & =\bar{f}(z(t), t)+(\bar{f}(x(t), t)-\bar{f}(z(t), t))+\mathbf{u} \\
+ & \frac{\eta}{2} \int_{t-2 / \eta}^{t} p(t, l) \mathrm{d} l \\
- & \frac{\eta}{2}\left\{\int_{t-2 / \eta}^{t} \int_{s}^{t}\left(\frac{\partial f}{\partial x}-\frac{\partial \bar{f}}{\partial x}\right) \mathrm{d} l \mathrm{~d} s\right\}(f+\mathbf{u})
\end{aligned}
$$

where we suppress the arguments $\left(x(t), l, \eta^{2} l\right)$ of $\partial f / \partial x$ and $(x(t), l)$ of $\partial \bar{f} / \partial x$ and the arguments of $f\left(x(t), t, \eta^{2} t\right)$ and $\mathbf{u}(t)$, whenever this would not lead to confusion.

Let $V, \delta_{1}$, and $\delta_{2}$ satisfy the requirements P1)-P3) from Definition 3 and (L1). By property P1) with $\xi=z(t)$ and (20), the derivative of $V(z, t)$ along the trajectories $z(t)$ (which we denote simply by $\dot{V}$ in the sequel) satisfies

$$
\begin{aligned}
\dot{V} \leq- & \bar{c} \delta^{2}(|z(t)|)+V_{\xi}(z(t), t)(\bar{f}(x(t), t)-\bar{f}(z(t), t)) \\
& +\frac{\eta}{2} V_{\xi}(z(t), t) \int_{t-2 / \eta}^{t} p(t, l) \mathrm{d} l \\
& -\frac{\eta}{2} V_{\xi}(z(t), t)\left[\int_{t-2 / \eta}^{t} \int_{s}^{t}\left(\frac{\partial f}{\partial x}-\frac{\partial \bar{f}}{\partial x}\right) \mathrm{d} l \mathrm{~d} s\right] \\
& \times(f+\mathbf{u})+V_{\xi}(z(t), t) \mathbf{u} .
\end{aligned}
$$

We deduce from (14)-(16), (19), and P2) that

$$
\begin{aligned}
\dot{V} \leq & -\bar{c} \delta^{2}(|z(t)|)+K \delta(|z(t)|)|x(t)-z(t)| \\
& +\frac{\eta}{2} \delta(|z(t)|)\left|\int_{t-2 / \eta}^{t} p(t, l) \mathrm{d} l\right|+\delta(|z(t)|)|\mathbf{u}| \\
& +\frac{\eta}{2}(|f|+|\mathbf{u}|) \delta(|z(t)|) \int_{t-2 / \eta}^{t} \int_{s}^{t} 2 K \mathrm{~d} l \mathrm{~d} s \\
\leq \quad & -\bar{c} \delta^{2}(|z(t)|)+K \delta(|z(t)|)|R(x(t), t)| \\
& +\frac{\eta}{2} \delta(|z(t)|) N(\eta) \delta(|x(t)| / 2)+\delta(|z(t)|)|\mathbf{u}| \\
& +\frac{2}{\eta} K \delta(|z(t)|)\{\delta(|x(t)| / 2)+|\mathbf{u}|\} .
\end{aligned}
$$

Moreover, (15), P2), and (19) give

$$
\begin{aligned}
|R(x(t), t)| & \leq \frac{\eta}{2} \int_{t-2 / \eta}^{t} \int_{s}^{t}|p(t, l)| \mathrm{d} l \mathrm{~d} s \\
& \leq \frac{2}{\eta} \delta(|x(t)| / 2) .
\end{aligned}
$$

Combining these inequalities and grouping terms gives

$$
\begin{aligned}
\dot{V} \leq & -\bar{c} \delta^{2}(|z(t)|)+\delta(|z(t)|)|\mathbf{u}| \\
& +\delta(|z(t)|)\left(\frac{4}{\eta} K+\frac{\eta}{2} N(\eta)\right)\{\delta(|x(t)| / 2)+|\mathbf{u}|\} .
\end{aligned}
$$

On the other hand, (16), (21), and P3) give

$$
\begin{aligned}
|z(t)| & \geq|x(t)|-|R(x(t), t)| \\
& \geq|x(t)|-\frac{\overline{\bar{c}}}{\eta}|x(t)| \geq \frac{1}{2}|x(t)|
\end{aligned}
$$

when $\eta \geq \max \left\{2 \overline{\bar{c}}, \eta_{o}\right\}$. Since $\delta \in \mathcal{K}$, this gives

$$
\begin{aligned}
\dot{V} \leq & \left(-\bar{c}+\frac{4}{\eta} K+\frac{\eta}{2} N(\eta)\right) \delta^{2}(|z(t)|) \\
& +\left(\frac{4}{\eta} K+\frac{\eta}{2} N(\eta)+1\right) \delta(|z(t)|)|\mathbf{u}| .
\end{aligned}
$$


Setting $\chi(s)=\frac{\bar{c}}{4} \delta(s / 2)$, it then follows from (22) that

$$
\begin{aligned}
& |\mathbf{u}|_{\infty} \leq \chi(|x(t)|) \quad \Rightarrow \quad|\mathbf{u}|_{\infty} \leq \chi(2|z(t)|) \\
& \Rightarrow \quad \dot{V} \leq\left(-\frac{3 \bar{c}}{4}+\frac{8}{\eta} K+\eta N(\eta)\right) \delta^{2}(|z(t)|) .
\end{aligned}
$$

Setting $V^{[\alpha]}(x, t):=V(x+R(x, t), t)$, we see the derivative $\dot{V}=V_{t}(z, t)+V_{\xi}(z, t) \dot{z}$ of $V(z, t)$ along (16) satisfies

$$
\dot{V}=V_{t}^{[\alpha]}(x, t)+V_{x}^{[\alpha]}(x, t)\{f(x, t, \alpha t)+\mathbf{u}\} .
$$

We deduce from property (5) of $N \in \mathcal{M}$, (22), and (24) that when the $\alpha$ 's (and so also the $\eta$ 's) are sufficiently large,

$$
\begin{aligned}
& |u| \leq \chi(|x|) \Rightarrow \\
& V_{t}^{[\alpha]}(x, t)+V_{x}^{[\alpha]}(x, t)[f(x, t, \alpha t)+u] \leq-\frac{\bar{c}}{2} \delta^{2}(|x| / 2)
\end{aligned}
$$

and $\delta_{1}(|x| / 2) \leq V^{[\alpha]}(x, t) \leq \delta_{2}(|x|+2 \delta(|x| / 2) / \eta)$ by (21). It follows that $V^{[\alpha]}$ is an ISS Lyapunov function for (13), so (13) is ISS for large $\alpha$, by Lemma 7, as claimed. The UGAS conclusion is the special case where $\mathbf{u} \equiv 0$. To prove the iISS assertion, we instead follow the preceding argument up through (23) (which is valid since that part did use the unboundedness of $\delta$ ) and then substitute the relation

$$
\delta(|z(t)|)|\mathbf{u}| \leq \frac{\bar{c}}{2} \delta^{2}(|z(t)|)+\frac{1}{2 \bar{c}}|\mathbf{u}|^{2}
$$

into (23) and bound the resulting coefficient of $|\mathbf{u}|$ to show that $V^{[\alpha]}$ is an iISS Lyapunov function for (13) for sufficiently large $\alpha$ (by again using (21) and (22) and taking $\nu(s)=\delta^{2}(s / 2) \bar{c} / 4$ and $\Delta(s)=\bar{r} s^{2}$ for a suitable $\left.\bar{r}>0\right)$, which implies that (13) is iISS for large $\alpha$, by Lemma 7 .

We turn next to the special case where (2) is globally exponentially stable. Let $V$ satisfy the requirements of Lemma 1 above for (2), and let $x(t)$ be any trajectory for (13) for any control $\mathbf{u} \in \mathcal{U}$ starting at $x\left(t_{0}\right)=x_{0}$. Define $z(t)$ by (16)-(17). Arguing as before except with this new $V$ gives $|R(x(t), t)| \leq \frac{2 K}{\eta}|x(t)|$ and (25) satisfies (by P3) and (14))

$$
\begin{aligned}
\dot{V} \leq & -|z(t)|^{2}+c_{3}|z(t)||\mathbf{u}| \\
& +c_{3}|z(t)|\left(\frac{4}{\eta} K^{2}+\frac{\overline{\bar{c}} \eta}{2} N(\eta)\right)\{|x(t)|+|\mathbf{u}|\} \\
\leq & \left(-1+\frac{8}{\eta} c_{3} K^{2}+\overline{\bar{c}} \eta c_{3} N(\eta)\right)|z(t)|^{2} \\
& +c_{3}|z(t)|\left(\frac{4}{\eta} K^{2}+\frac{\bar{c} \eta}{2} N(\eta)+1\right)|\mathbf{u}|,
\end{aligned}
$$

since $|x(t)| \leq 2|z(t)|$ for large $\eta$ as before. If we now define $\tilde{\chi} \in \mathcal{K}_{\infty}$ by $\tilde{\chi}(s)=s /\left\{8\left(1+c_{3}\right)\right\}$, then we deduce as in the UGAS case that if $\eta$ is large enough, and if $|\mathbf{u}|_{\infty} \leq$ $\tilde{\chi}(|x(t)|)$ for all $t$, then also $|\mathbf{u}|_{\infty} \leq \tilde{\chi}(2|z(t)|)$ for all $t$ and $\dot{V} \leq-|z(t)|^{2} / 2 \leq-V(z(t), t) /\left(2 c_{2}\right)$. This gives the decay $V(z(t), t) \leq V\left(z\left(t_{0}\right), t_{0}\right) e^{-\left(t-t_{o}\right) /\left(2 c_{2}\right)}$ (when $\left.t \geq t_{o}\right)$, so

$\frac{c_{1}}{4}|x(t)|^{2} \leq c_{1}|z(t)|^{2} \leq V(z(t), t) \leq c_{2}\left|z\left(t_{0}\right)\right|^{2} e^{-\frac{t-t_{o}}{2 c_{2}}}$,

so our estimate on $|R(x(t), t)|$ and the form of $z(t)$ give

$$
\begin{aligned}
|x(t)| & \leq \sqrt{\frac{4 c_{2}}{c_{1}}}\left|x\left(t_{0}\right)+R\left(x\left(t_{0}\right), t_{0}\right)\right| e^{-\frac{1}{4 c_{2}}\left(t-t_{o}\right)} \\
& \leq \sqrt{\frac{4 c_{2}}{c_{1}}}\left(1+\frac{2 K}{\eta}\right)\left|x\left(t_{0}\right)\right| e^{-\frac{1}{4 c_{2}}\left(t-t_{o}\right)} .
\end{aligned}
$$

We conclude as before that if (2) is UGES, then, when $\alpha>0$ is large enough, (1) is also UGES and (by the proof of [16, Lemma 2.14]) (13) is ISES, proving our theorem.
The preceding proof provides the following construction:

Corollary 10: Let the hypotheses of Theorem 8 hold for some $\delta \in \mathcal{K}$ and $V \in C^{1}$ be a Lyapunov function for (2) satisfying the $\delta$-compatibility requirements for (2). Then there is a constant $\underline{\alpha}>0$ such that for each constant $\alpha>\underline{\alpha}$,

$$
\begin{aligned}
& V^{[\alpha]}(\xi, t):= \\
& V\left(\xi-\frac{\sqrt{\alpha}}{2} \int_{t-\frac{2}{\sqrt{\alpha}}}^{t} \int_{s}^{t}\{f(\xi, l, \alpha l)-\bar{f}(\xi, l)\} \mathrm{d} l \mathrm{~d} s, t\right)
\end{aligned}
$$

is a Lyapunov function for (1) and an iISS Lyapunov function for (13). If in addition $\delta \in \mathcal{K}_{\infty}$, then $V^{[\alpha]}$ is also an ISS Lyapunov function for (13).

Remark 11: The decay requirement (5) on $N \in \mathcal{M}$ from Theorem 8 can be relaxed, as follows. We assume the flow map $\phi$ of the limiting dynamics (2) satisfies the UGES conditions (6)-(7) for some $D>1$ and $\lambda \in(0, K)$, where $K$ satisfies (15), and we let $V$ be as in Lemma 1. By Remark 2 , our argument above shows that Theorem 8 remains true for UGES $\bar{f}$ if its condition $N \in \mathcal{M}$ is relaxed to

$$
\exists \eta^{\star}>0 \text { s.t. } \sup _{\eta \geq \eta^{\star}} \eta N(\eta)<\frac{K-\lambda}{11 D(\Theta-1) \overline{\bar{c}}} .
$$

A similar relaxation can be made in the more general UGAS setting covered by our theorem.

Remark 12: The method we used in the proof of Theorem 8 can be used to prove the ISS property for (3) under appropriate growth assumptions on the matrix-valued function $g: \mathbb{R}^{n} \times \mathbb{R} \times \mathbb{R} \rightarrow \mathbb{R}^{n \times m}$. Clearly, some growth condition on $g$ is needed and linear growth of $g$ is not enough, since

$$
\dot{x}=-x+x u
$$

is not ISS. One way to extend our theorem to (3) is to add the hypothesis that $g$ is $C^{1}$ and that there is a constant $c_{o}>1$ such that for all $t \in \mathbb{R}, x \in \mathbb{R}^{n}$, and $\alpha>0$,

$$
\|g(x, t, \alpha t)\| \leq c_{o}+\sqrt{\delta(|x| / 2)}
$$

where $\|\cdot\|$ is the 2-norm on $\mathbb{R}^{n \times m}$ and $\delta \in \mathcal{K}_{\infty}$ satisfies P1)-P3) for some Lyapunov function $V$. Applying the first part of the proof of Theorem 8 except with the new choice

$$
\chi(s)=\frac{\bar{c} \delta(s / 2)}{4\left\{c_{o}+\sqrt{\delta(s / 2)}\right\}},
$$

we then conclude as before that (3) is ISS for sufficiently large $\alpha>0$ (with the same ISS Lyapunov function $V^{[\alpha]}$ ). If instead $\delta \in \mathcal{K}$ is bounded, then (3) is iISS when $\alpha$ is sufficiently large, by our earlier argument.

\section{ILLUSTRATIONS}

The following applications show how our results extend the known results [7], [12]. In [12], the limiting dynamics (2) are assumed to be UGES. However, in our first example, the limiting dynamics are UGAS but not necessarily UGES. We also apply our results to a friction model for a mass-spring dynamics. In both examples, the limiting dynamics has a simple explicit Lyapunov function so our constructions give explicit Lyapunov functions for the original dynamics. 


\section{A. Application To a UGAS Dynamic That Is Not UGES}

Consider this variant of the scalar example on [12, p.53]:

$$
\begin{aligned}
& \dot{x}=f(x, t, \alpha t)= \\
& -\sigma_{1}(x)\left[2+\sin \left(t+\cos \left(\sigma_{2}(x)\right)\right)\right]\{1+10 \sin (\alpha t)\}
\end{aligned}
$$

where $\sigma_{1}, \sigma_{2}: \mathbb{R} \rightarrow \mathbb{R}$ are $C^{1}$ functions such that

$\sigma_{1}$ is odd, $\sup \left\{\left|\sigma_{1}^{\prime}(x)\right|+\left|\sigma_{1}(x) \sigma_{2}^{\prime}(x)\right|: x \in \mathbb{R}\right\}<\infty$, $\sigma_{1} \in \mathcal{K}$ on $[0, \infty)$, and $\sigma_{1}^{\prime \prime}(s) \leq 0 \forall s>0$.

Using simple calculations, one checks the assumptions of Theorem 8 using

$$
\begin{aligned}
& \bar{f}(x, t):=-\sigma_{1}(x)\left[2+\sin \left(t+\cos \left(\sigma_{2}(x)\right)\right)\right] \\
& V(x, t) \equiv \bar{V}(x):=\int_{0}^{x} \sigma_{1}(s) \mathrm{d} s, \\
& \delta(s):=33 \sigma_{1}(2 s), \text { and } N(\eta):=60 / \eta^{2} \text { for large } \eta .
\end{aligned}
$$

This allows e.g. $\sigma_{1}(s)=\sigma_{2}(s)=\arctan (s)$ in which case (2) is UGAS but not UGES because $|\dot{x}(t)| \leq 2 \pi$ along all of its trajectories $x(t)$ and all $t \geq 0$. Condition P1) follows because $\sigma_{1}(2 s) \leq 2 \sigma_{1}(s)$ for all $s \geq 0$, which holds because $\sigma_{1}^{\prime \prime}(s) \leq 0$ for all $s \geq 0$. Corollary 10 then gives the following iISS Lyapunov function for (13) for large $\alpha>0$ :

$$
\bar{V}\left(\xi+5 \sqrt{\alpha} \sigma_{1}(\xi) \int_{t-\frac{2}{\sqrt{\alpha}}}^{t} \int_{s}^{t} \mu(\xi, l) \sin (\alpha l) \mathrm{d} l \mathrm{~d} s\right),
$$

where $\mu(\xi, l):=2+\sin \left(l+\cos \left(\sigma_{2}(\xi)\right)\right)$. In particular, this is a Lyapunov function for $\dot{x}=f(x, t, \alpha t)$, and it is also an ISS Lyapunov function for (13) if $\delta \in \mathcal{K}_{\infty}$. Our conditions on the $\sigma_{i}$ 's cannot be omitted even if (2) is UGES [12, §8.2]. For example, if $\sigma_{1}(x)=x$ and $\sigma_{2}(x)=x^{2}$, then (2) is UGES, but (29) is only shown to be locally exponentially stable for large $\alpha>0$ [12]. This does not contradict our theorem because in that case (15) would be violated.

\section{B. Friction Example}

The following dynamical system arises in the control of mechanical systems in the presence of friction. We consider the one degree-of-freedom mass-spring system [3]

$$
\begin{aligned}
\dot{x}_{1}= & x_{2} \\
\dot{x}_{2}= & -\sigma_{1}(\alpha t) x_{2}-k(t) x_{1}+u \\
& -\left\{\sigma_{2}(\alpha t)+\sigma_{3}(\alpha t) e^{-\beta_{1} \mu\left(x_{2}\right)}\right\} \operatorname{sat}\left(x_{2}\right)
\end{aligned}
$$

where $x_{1}$ and $x_{2}$ are the mass position and velocity, respectively; $\sigma_{i}, i=1,2,3$ denote positive time-varying viscous, Coulomb, and static friction-related coefficients, respectively; $\beta_{1}$ is a positive constant corresponding to the Stribeck effect; $\mu(\cdot)$ is a positive definite function also related to the Stribeck effect; $k$ denotes a positive time-varying spring stiffness-related coefficient; and sat $(\cdot)$ denotes any continuous function having these properties:

$$
\begin{aligned}
& \text { (a) } \operatorname{sat}(0)=0, \quad \text { (b) } \xi \operatorname{sat}(\xi) \geq 0 \quad \forall \xi \in \mathbb{R}, \\
& \text { (c) } \lim _{\xi \rightarrow+\infty} \operatorname{sat}(\xi)=+1, \quad \text { (d) } \lim _{\xi \rightarrow-\infty} \operatorname{sat}(\xi)=-1
\end{aligned}
$$

Here, we model the saturation differentiably as

$$
\operatorname{sat}\left(x_{2}\right)=\tanh \left(\beta_{2} x_{2}\right),
$$

where $\beta_{2}$ is a large positive constant. Note for later use that $\left|\operatorname{sat}\left(x_{2}\right)\right| \leq \beta_{2}\left|x_{2}\right|$ for all $x_{2} \in \mathbb{R}$. We assume the friction coefficients vary in time faster than the spring stiffness coefficient so we restrict to cases where $\alpha>1$.

Our precise assumptions on (31) are: $k$ and the $\sigma_{i}$ 's are bounded $C^{1}$ functions; $\mu$ has a globally bounded derivative; and there exist a function $M: \mathbb{R}_{\geq 0} \rightarrow \mathbb{R}_{\geq 0}: s \mapsto M(s)$ that is $o(s)$ (i.e. $M(s) / s \rightarrow 0$ as $s \rightarrow+\infty)$ and constants $\tilde{\sigma}_{i}$, with $\tilde{\sigma}_{1}>0$ and $\tilde{\sigma}_{i} \geq 0$ for $i=2,3$, such that

$$
\left|\int_{t_{1}}^{t_{2}}\left(\sigma_{i}(t)-\tilde{\sigma}_{i}\right) \mathrm{d} t\right| \leq M\left(t_{2}-t_{1}\right), \quad i=1,2,3
$$

for all $t_{1}, t_{2} \in \mathbb{R}$ satisfying $t_{2}>t_{1}$. Although the $\sigma_{i}$ 's are positive for physical reasons, we will not require their positivity in the sequel. Clearly, (34) holds for constant positive $\sigma_{i}$ 's using $\sigma_{i} \equiv \tilde{\sigma}_{i}$, but (34) also allows the $\sigma_{i}$ 's to take negative values on intervals of arbitrarily large length; see Remark 14 below. We show (31) satisfies the requirements of the version of our theorem from Remark 12 (with $\delta$ of the form $\delta(s)=\bar{r} s$ ) when (2) is given by

$$
\begin{aligned}
\dot{x}_{1}= & x_{2} \\
\dot{x}_{2}= & -\tilde{\sigma}_{1} x_{2}-\left\{\tilde{\sigma}_{2}+\tilde{\sigma}_{3} e^{-\beta_{1} \mu\left(x_{2}\right)}\right\} \operatorname{sat}\left(x_{2}\right) \\
& -k(t) x_{1},
\end{aligned}
$$

assuming the following additional condition whose physical interpretation is that the spring stiffness is nonincreasing:

$$
\exists k_{o}, \bar{k}>0 \text { s.t. } k_{o} \leq k(t) \leq \bar{k} \text { and } k^{\prime}(t) \leq 0 \quad \forall t \geq 0 .
$$

To this end, set $S:=\tilde{\sigma}_{1}+\left(\tilde{\sigma}_{2}+\tilde{\sigma}_{3}\right) \beta_{2}$ and

$$
V(x, t)=A\left(k(t) x_{1}^{2}+x_{2}^{2}\right)+x_{1} x_{2},
$$

where $A:=1+1 / k_{o}+\left[1+S^{2} / k_{o}\right] / \tilde{\sigma}_{1}$. Since $A \bar{k} \geq 1$,

$$
\frac{1}{2}\left(x_{1}^{2}+x_{2}^{2}\right) \leq V(x, t) \leq A^{2} \bar{k}\left(\left|x_{1}\right|+\left|x_{2}\right|\right)^{2}
$$

for all $x \in \mathbb{R}^{2}$ and $t \geq 0$. Also, since $k^{\prime} \leq 0$ everywhere, the derivative $\dot{V}=V_{t}(x, t)+V_{x}(x, t) \bar{f}(x, t)$ of $V$ along trajectories of (35) satisfies

$$
\begin{aligned}
\dot{V} \leq & V_{x}(x, t) \bar{f}(x, t)=\left[2 A k(t) x_{1}+x_{2}\right] x_{2}-\left[2 A x_{2}+x_{1}\right] \\
& \times\left\{\tilde{\sigma}_{1} x_{2}+\left[\tilde{\sigma}_{2}+\tilde{\sigma}_{3} e^{-\beta_{1} \mu\left(x_{2}\right)}\right] \operatorname{sat}\left(x_{2}\right)+k(t) x_{1}\right\}
\end{aligned}
$$

and therefore, by grouping terms, we also have

$$
\begin{aligned}
& \dot{V} \leq-k_{0} x_{1}^{2}-\left(2 A \tilde{\sigma}_{1}-1\right) x_{2}^{2}+S\left|x_{1} x_{2}\right| \quad(\text { by }(32)(\mathrm{b})) \\
& \leq-b|x|^{2}-\left[\frac{k_{o}}{2} x_{1}^{2}+\left(A \tilde{\sigma}_{1}-1 / 2\right) x_{2}^{2}-S\left|x_{1} x_{2}\right|\right] \\
& =-b|x|^{2}-\frac{k_{o}}{2}\left(\left|x_{1}\right|-\frac{S}{k_{o}}\left|x_{2}\right|\right)^{2}+\left(\frac{S^{2}}{2 k_{o}}+\frac{1}{2}-A \tilde{\sigma}_{1}\right) x_{2}^{2} \\
& \leq-b|x|^{2}, \text { where } b:=\min \left\{k_{o} / 2, A \tilde{\sigma}_{1}-1 / 2\right\} .
\end{aligned}
$$

The preceding inequalities imply that $V / b$ is a Lyapunov function for (35) satisfying the requirements of Lemma 1. The integral bound requirement (14) from our theorem follows from (34) and the sublinear growth of tanh, since the integral bound can be verified term by term. We conclude from our earlier construction that for $\alpha>0$ sufficiently large, (31) admits the ISS Lyapunov function

$$
V^{[\alpha]}(\xi, t)=V\left(\xi_{1}, \xi_{2}+\frac{\sqrt{\alpha}}{2} \int_{t-\frac{2}{\sqrt{\alpha}}}^{t} \int_{s}^{t} \Gamma_{\alpha}(l, \xi) \mathrm{d} l \mathrm{~d} s, t\right)
$$


where $V$ is the Lyapunov function (36) for (35) and where

$$
\begin{array}{r}
\Gamma_{\alpha}(l, \xi):=\left\{\sigma_{1}(\alpha l)-\tilde{\sigma}_{1}\right\} \xi_{2}+\mu_{\alpha}(l, \xi) \tanh \left(\beta_{2} \xi_{2}\right) \\
\mu_{\alpha}(l, \xi):=\sigma_{2}(\alpha l)-\tilde{\sigma}_{2}+\left(\sigma_{3}(\alpha l)-\tilde{\sigma}_{3}\right) e^{-\beta_{1} \mu\left(\xi_{2}\right)}
\end{array}
$$

so (31) is ISS for large enough $\alpha>0$, by Remark 12 .

Remark 13: The preceding construction simplifies considerably if $\sigma_{2}$ and $\sigma_{3}$ in (31) are positive constants. In that case, the limiting dynamics (2) can be taken to be

$$
\begin{aligned}
& \dot{x}_{1}=x_{2} \\
& \dot{x}_{2}=-\tilde{\sigma}_{1} x_{2}-\left\{\sigma_{2}+\sigma_{3} e^{-\beta_{1} \mu\left(x_{2}\right)}\right\} \operatorname{sat}\left(x_{2}\right)-k(t) x_{1}
\end{aligned}
$$

and the ISS Lyapunov function for (31) becomes

$$
V\left(\xi_{1}, \xi_{2}\left(1+\frac{\sqrt{\alpha}}{2} \int_{t-\frac{2}{\sqrt{\alpha}}}^{t} \int_{s}^{t}\left\{\sigma_{1}(\alpha l)-\tilde{\sigma}_{1}\right\} \mathrm{d} l \mathrm{~d} s\right), t\right)
$$

with $V$ defined by (36), since the $\mu_{\alpha}(l, \xi) \tanh \left(\beta_{2} \xi_{2}\right)$ term in the difference $f-\bar{f}$ is no longer present in the CLF.

Remark 14: We show that hypothesis (34) with $\tilde{\sigma}_{1}>0$ allows $\sigma_{1}$ to take negative values on intervals of arbitrarily large length. We first introduce the sets $\mathcal{D}=\left\{ \pm 2^{j}: j \in \mathbb{N}\right\}$, $J=\left\{s \in \mathbb{R}: \exists j \in \mathbb{N}\right.$ s.t. $\left.|s| \in\left[2^{j}, 2^{j}+1\right]\right\}$, and the intervals $I_{j}^{-}:=\left[2^{j}-2^{-j}, 2^{j}\right]$ and $I_{j}^{+}=\left[2^{j}+1,2^{j}+2^{-j}+1\right]$ for $j \in \mathbb{N}$. Define the even continuous function

$$
\sigma_{1}(s):= \begin{cases}-1, & s \in J \\ -2^{j+1}\left(|s|-2^{j}\right)-1, & |s| \in I_{j}^{-}, j \in \mathbb{N} \\ 2^{j+1}\left(|s|-2^{j}-1\right)-1, & |s| \in I_{j}^{+}, j \in \mathbb{N} \\ 1, & \text { otherwise }\end{cases}
$$

Then $\sigma_{1} \equiv 1$ outside a small neighborhood of $J$, and $\sigma_{1}$ is affine on the intervals $I_{j}^{ \pm}$.

Using the indicator function $\chi_{J}$ (defined to be 1 on $J$ and 0 otherwise) and Lebesgue measure $\lambda$, and letting $\mathcal{I}$ denote the set of all intervals in $\mathbb{R}$, we get

$$
\int_{-\infty}^{\infty}\left(1-\chi_{J}\right)\left|\sigma_{1}(s)-1\right| \mathrm{d} s=4 \sum_{j=1}^{\infty} 2^{-j}=4
$$

so the left side of (34) with $i=1$ and $\tilde{\sigma}_{1}=1$ is at most

$$
\begin{aligned}
& 2 \int_{t_{1}}^{t_{2}} \chi_{J}(s) \mathrm{d} s+4=2 \lambda\left(\left[t_{1}, t_{2}\right] \cap J\right)+4 \leq \\
& 2 \sup _{I \in \mathcal{I}}\left\{\lambda(I \cap J): \lambda(I) \leq t_{2}-t_{1}\right\}+4=: M\left(t_{2}-t_{1}\right) .
\end{aligned}
$$

With $M$ so defined and \# denoting cardinality, we have

$$
\begin{aligned}
\frac{M(s)}{s} & =\frac{2}{s}\left\{2+\sup _{I \in \mathcal{I}}\{\lambda(I \cap J): \lambda(I) \leq s\}\right\} \\
& \leq \frac{2}{s}\left\{2+\sup _{I \in \mathcal{I}}\{\#\{I \cap \mathcal{D}\}: \lambda(I) \leq s\}\right\} \\
& \leq \frac{4}{s}\left\{2+\log _{2}(s+1)\right\} \rightarrow 0 \text { as } s \rightarrow+\infty
\end{aligned}
$$

(since for any $I \in \mathcal{I}$ with $\lambda(I) \leq s$, and any $l, r \in \mathbb{N}$, if $2^{r}, 2^{r+1}, \ldots, 2^{r+l} \in \mathcal{D} \cap I$, then $2^{r+l}-2^{r} \leq s$, hence $l \leq \log _{2}\left(s 2^{-r}+1\right) \leq \log _{2}(s+1)$, which gives the inequality in (40)), so $\sigma_{1}$ satisfies (34) with $\tilde{\sigma}_{1}=1$.

\section{CONCLUSION}

We showed that assumptions similar to those of [12, Theorem 3] are sufficient for global (rather than just local) exponential stability of rapidly time-varying nonlinear systems. Further, we extended [7], [12] by establishing global asymptotic (but not necessarily exponential) stability of the fast time-varying dynamics under milder conditions on the limiting dynamics, and by constructing strict Lyapunov functions for fast time-varying systems in terms of Lyapunov functions for the limiting dynamics. Our results complement those of [12]. Our Lyapunov function constructions are new even in the special case where the dynamics are exponentially stable, and are also input-to-state stable Lyapunov functions when the fast-varying dynamics are control affine, under appropriate conditions on the vector fields defining the systems. We illustrated the applicability of our methods using a friction control example.

\section{REFERENCES}

[1] D. Angeli, E.D. Sontag, and Y. Wang, A Characterization of Integral Input to State Stability, IEEE Trans. Automatic Control, vol. 45, 2000 , pp. 1082-1097.

[2] A. Bacciotti and L. Rosier, Liapunov Functions and Stability in Control Theory, 2nd Edition, Springer Verlag, New York; 2005.

[3] M.S. de Queiroz, D.M. Dawson, S. Nagarkatti, and F. Zhang, Lyapunov-Based Control of Mechanical Systems, Birkhäuser, Cambridge, MA; 2000.

[4] H. Edwards, Y. Lin, and Y. Wang, "On Input-to-State Stability for Time-Varying Nonlinear Systems", in Proc. 39th IEEE Conf. Decis. Control, Sydney, Australia, 2000, pp. 3501-3506.

[5] L. Gruene, E.D. Sontag, and F. Wirth, Asymptotic Stability Equals Exponential Stability, and ISS Equals Finite Energy Gain - If You Twist Your Eyes, Systems Control Lett., vol. 38, 1999, pp. 127-134.

[6] J.K. Hale, Ordinary Differential Equations, Krieger, Malabor, FL; 1980.

[7] H. Khalil, Nonlinear Systems, Third Edition, Prentice Hall, Englewood Cliffs, NJ; 2002.

[8] Y. Lin, Y. Wang, and D. Cheng, "On nonuniform and semi-uniform input-to-state stability for time varying systems", in Proceedings of the 16th IFAC World Congress (Praha 2005), to appear.

[9] M. Malisoff and F. Mazenc, "Further Constructions of Strict Lyapunov Functions for Time-Varying Systems", in Proceedings of the American Control Conference, Portland, OR, 2005, pp. 1889-1894.

[10] M. Malisoff and F. Mazenc, Further Remarks on Strict Input-to-State Stable Lyapunov Functions for Time-Varying Systems, Automatica, vol. 41, 2005, pp. 1973-1978.

[11] J. Peuteman and D. Aeyels, Averaging Results and the Study of Uniform Asymptotic Stability of Homogeneous Differerential Equations that are Not Fast Time-Varying, SIAM J. Control Optim., vol. 37, 1999, pp. $997-1010$

[12] J. Peuteman and D. Aeyels, Exponential Stability of Nonlinear TimeVarying Differential Equations and Partial Averaging, Mathematics of Control, Signals, and Systems, vol. 15, no. 1, 2002, pp. 42-70.

[13] J. Peuteman and D. Aeyels, Exponential Stability of Slowly TimeVarying Nonlinear Systems, Mathematics of Control, Signals, and Systems, vol. 15, no. 3, 2002, pp. 202-228.

[14] E.D. Sontag, Smooth Stabilization Implies Coprime Factorization, IEEE Trans. Automatic Control, vol. 34, 1989, pp. 435-443.

[15] E.D. Sontag, Comments on Integral Variants of ISS, Systems Control Lett., vol. 34, 1998, pp. 93-100.

[16] E.D. Sontag and Y. Wang, On Characterizations of the Input-to-State Stability Property, Systems Control Lett., vol. 24, 1995, pp. 351-359.

[17] J. Tsinias, A General Notion of Global Asymptotic Controllability for Time-Varying Systems and Its Lyapunov Characterization, International Journal of Control, vol. 78, 2005, pp. 264-276.

[18] J. Tsinias, Links Between Asymptotic Controllability and Persistence of Excitation for a Class of Time-Varying Systems, Systems Control Lett., vol. 54, 2005, pp. 1051-1062. 